\title{
FVIla Neutralization by a FXa Inhibitor: How It Could Dampen Tumorigenic Cancer Cell Phenotypes
}

\author{
Bernd Engelmann ${ }^{1}$ \\ ${ }^{1}$ Institut für Laboratoriumsmedizin, Klinikum der Universität \\ München, Ludwig-Maximilians-Universität, Munich, Germany \\ Thromb Haemost 2019;119:1383.
}

Pathological activation of coagulation contributes to the high incidence of venous thromboembolism in different types of cancer, in particular, gastrointestinal tumors. Besides this, single coagulation factors are able to promote cancer development by direct effects on tumor cell signaling mechanisms. One of the best described coagulation components implicated in tumor growth and metastasis is tissue factor (TF), which can be expressed by various tumor cells. TF in complex with factor VIIa (FVIIa) initiates the extrinsic arm of fibrin formation and, independently, activates cell signaling via protease activated receptors (PARs). ${ }^{1}$ The thoroughly conducted study by Featherby et $\mathrm{al}^{2}$ adds a new layer to the coagulation-cancer cell connection by highlighting the role of FVIIa both for the release of $\mathrm{TF}^{+}$microvesicles (MVs) and for tumor cell proliferation, as well as the inhibition of these effects by distinct direct oral anticoagulants. MVs are a species of extracellular vesicles that might support tumor progression by various mechanisms such as, for example, through delivery of their RNA cargo into the immune cell cytosol via the scavenger receptor CD36. ${ }^{3}$ PAR2 activation by FVIIa is suggested to be responsible for the enhancement of MV secretion and tumor cell proliferation. ${ }^{2}$ The direct FXa inhibitor apixaban, but not the FXa inhibitor rivaroxaban, decreases the proteolytic activity of FVIla and, consequently, the signaling function of PAR2 in cell prolifera-

\begin{abstract}
Address for correspondence Bernd Engelmann, MD, PhD, Institut für Laboratoriumsmedizin, Klinikum der Universität München, Ludwig-Maximilians-Universität, Munich, Germany (e-mail: Bernd.Engelmann@med.uni-muenchen.de).
\end{abstract}

tion. Based on calculations of the binding energies for the apixaban-FVIIa interaction, the authors suggest that the affinity of apixaban for FVIIa is in a comparable range as its affinity for FXa. By inhibiting cancer cell proliferation, the release of distinct extracellular vesicles, and coagulation activation per se, some FXa inhibitors could potentially target synchronously several different mechanisms of tumor progression. Such conclusions will require confirmation from additional work, especially from in vivo experiments.

Conflict of Interest

None declared.

\section{References}

1 Zelaya H, Rothmeier AS, Ruf W. Tissue factor at the crossroad of coagulation and cell signaling. J Thromb Haemost 2018;16(10): 1941-1952

2 Featherby S, Madkhali Y, Maraveyas A, Ettelaie C. Apixaban suppresses the release of TF-positive microvesicles and restrains cancer cell proliferation through directly inhibiting TF-fVIIa activity. Thromb Haemost 2019;119(09):1419-1432

3 Pfeiler S, Thakur M, Grünauer P, et al. CD36-triggered cell invasion and persistent tissue colonization by tumor microvesicles during metastasis. FASEB J 2019;33(02):1860-1872

\section{received}

July 31, 2019

accepted after revision

July 31, 2019 (c) 2019 Georg Thieme Verlag KG Stuttgart · New York
DOI https://doi.org/

$10.1055 / \mathrm{s}-0039-1695732$. ISSN 0340-6245. 